\title{
TESTING SERIAL INDEPENDENCE USING THE SAMPLE DISTRIBUTION FUNCTION
}

\author{
By Miguel A. Delgado
}

Universidad Carlos III de Madrid

\begin{abstract}
This paper presents and discusses a nonparametric test for detecting serial dependence. We consider a Cramèr-von Mises statistic based on the difference between the joint sample distribution and the product of the marginals. Exact critical values can be approximated from the asymptotic null distribution, or by resampling, randomly permuting the original series. A Monte Carlo experiment illustrates the test performance with small sample sizes. The paper also includes an application, testing the random walk hypothesis of exchange rate returns for several currencies.
\end{abstract}

Keywords. Serial independence tests; multivariate sample distribution; HoeffdingBlum-Kiefer-Rosenblatt empirical process; random permutation test; ergodic alternatives.

\section{INTRODUCTION}

Reasons for testing serial independence are available in generous supply. Although testing for lack of correlation has long been of interest, in a nonGaussian context statistical independence may be the relevant hypothesis to test. Inferences on probability models are often only valid under the assumption that observations form a random sample. In time series, it is not always obvious whether a pattern is present in the data. Serially dependent processes can exhibit zero autocorrelation, and evidence on the existence of subtle nonlinear dependence is provided by testing independence rather than lack of autocorrelation. This is the case with certain economic variables. For instance, there is evidence of non-Gaussian behavior in some financial time series, and a test of serial independence, rather than serial autocorrelatedness, on the rates of return (first differences in $\operatorname{logs}$ ) is an appropriate way of testing the random walk hypothesis.

Traditionally, serial independence has indeed been tested by means of the sample correlation coefficient, or, in Gaussian cases, the Lagrange multiplier principle. There are also a number of nonparametric tests based on ranks, e.g. the runs test, the turning point test and the Spearman-Wald-Wolfowitz serial rank correlation coefficient test. These procedures have been applied to time series problems by Knoke (1977), Bartels (1982), Dufour (1981), Hallin et al. 
(1985), Hallin and Puri (1988) and Hallin and Mélard (1988), to mention only a few.

The above mentioned test procedures work well under commonly used dependence structures, like ARMA models. However, the usual serial correlation or serial rank-based procedures fail to detect subtle nonlinear underlying dependence structures.

A number of independence tests have been constructed based on the fact that the null hypothesis holds if and only if the joint distribution equals the product of the marginals. In these procedures, the test statistic is a distance between the estimated joint distribution (or joint density) and the product of the estimated marginal distributions (or marginal densities). Hoeffding (1948) and Blum et al. (1961) proposed to estimate the joint and marginal distributions by the empirical distribution function, and introduced statistics based on the $L_{2}$ and $L_{\infty}$ distances. Rosenblatt (1975) (see also Nadaraya, 1989, Ch. 3.5) and Robinson (1991) used smooth kernel estimates of the densities. Rosenblatt's statistic is based on the $L_{2}$ distance and has a $\chi^{2}$ limiting null distribution; Robinson's is based on the Kullback-Leibler information criterion and achieves a normal asymptotic null distribution. Only Robinson proved consistency in a time series context. Recently, Chan and Tran (1992) have proposed to estimate the joint and marginal densities by the histogram, and have introduced a statistic based on the $L_{1}$ distance. They did not obtain the asymptotic null distribution, but they proved consistency under general alternatives. They implemented a permutation test. Procedures based on the kernel and histogram methods share the disadvantage of depending on the choice of a smoothing number. Statistics based on the empirical distribution function, or the empirical characteristic function, avoid estimation by smoothing procedures, but the limiting null distribution is not standard. Brock et al. (1987) developed a test based on concepts that arise in the theory of chaotic processes. The consistency of this test depends on the choice of a kernel and smoothing number (see the discussion in Robinson, 1991). Pinkse (1993) has proposed a statistic based on the squared difference of the empirical characteristic function and the product of the marginals, weighted by a suitable kernel function. This procedure depends also on the choice of a kernel and the statistic achieves a limiting $\chi^{2}$ distribution under the null hypothesis.

This paper proposes and discusses a serial independence test based on the empirical distribution function, which is consistent in the direction of a wide range of serial dependence structures. We apply the Hoeffding-Blum-KieferRosenblatt (HBKR) statistic (Hoeffding, 1948; Blum et al. 1961) to test serial independence. Skaug and Tjøstheim (1993) have shown that the asymptotic null distribution of this statistic, when testing first-order serial independence (one lag), is the same as that of the HBKR statistic used for testing independence of two random variables, which was tabulated by Blum et al. (1961). They also proposed a combination of these statistics for testing pairwise serial independence of several lags. We derive the asymptotic distribution in the higher dimensional case, which differs from HBKR in a nonobvious way. We 
also propose a permutation test. Reported simulations support that the test enjoys good power and size properties in small samples.

The rest of the paper is organized as follows. Section 2 presents the statistics. Section 3 discusses asymptotic properties under the null and fixed alternatives. Section 4 presents and justifies a resampled version of the test. Section 5 summarizes a simulation study and an empirical application of the test procedure to testing serial independence using daily, monthly and quarterly data on pound, yen and deutschmark exchange rate changes. A final section is devoted to conclusions. Proofs are confined to an Appendix.

\section{THE TEST STATISTIC}

Let $\left\{X_{1}, X_{2}, \ldots, X_{T+p}\right\}$ be $T+p$ observations of the real-valued strictly stationary process $\left\{X_{t}\right\}$. Let $Z_{t}=\left(X_{t}, X_{t+1}, \ldots, X_{t+p}\right)$. Assume that $Z_{t}$ has a continuous distribution. We test serial $p$ dependence by testing

$$
\mathrm{H}_{0}: S(x)=0 \text { for all } x=\left(x_{1}, \ldots, x_{p+1}\right) \in \mathbb{R}^{p+1}
$$

versus

$$
\mathrm{H}_{1}: S(x) \neq 0 \text { for some } x=\left(x_{1}, \ldots, x_{p+1}\right) \in \mathbb{R}^{p+1}
$$

where

$$
S(x)=F(x)-\prod_{j=1}^{p+1} F_{1}\left(x_{j}\right)
$$

and

$$
F(x)=\operatorname{Pr}\left(X_{1} \leqslant x_{1}, X_{2} \leqslant x_{2}, \ldots, X_{p+1} \leqslant x_{p+1}\right), \quad F_{1}\left(x_{i}\right)=\operatorname{Pr}\left(X_{1} \leqslant x_{i}\right) .
$$

The null hypothesis states that $\left(X_{t}, X_{t+1}, \ldots, X_{t+p}\right)$ are completely independent.

Note that,

$$
S(x)=E\left\{\prod_{j=1}^{p+1} 1\left(X_{j} \leqslant x_{j}\right)\right\}-\prod_{j=1}^{p+1} E\left\{1\left(X_{j} \leqslant x_{j}\right)\right\}
$$

where $1(A)$ is the indicator function of the event $A$. Then, the sample analog of $S(x)$ is

$$
S_{T}(x)=T^{-1} \sum_{t=1}^{T} \prod_{j=1}^{p+1} 1\left(X_{t+j-1} \leqslant x_{j}\right)-\prod_{j=1}^{p+1}\left\{T^{-1} \sum_{t=1}^{T} 1\left(X_{t+j-1} \leqslant x_{j}\right)\right\} .
$$

Since $S_{T}(x)$ takes small values under $\mathrm{H}_{0}$ and larger values under $\mathrm{H}_{1}$, it forms a basis for testing $\mathrm{H}_{0}$. We consider the statistic 


$$
B_{T}=\sum_{t=1}^{T} S_{T}\left(Z_{t}\right)^{2}
$$

constructed in the spirit of Cramér-von Mises. This test statistic was first introduced by Hoeffding (1948) and Blum et al. (1961) for testing complete independence of a set of random variables from a random sample. Note also that, unlike alternative procedures based on densities, one does not need to choose a smoothing number.

\section{ASYMPTOTIC PROPERTIES OF $\boldsymbol{B}_{T}$}

Let $F_{1}^{-1}($.$) be the inverse function of the marginal distribution F_{1}($.$) ,$ $x_{i}=F_{1}^{-1}\left(\omega_{i}\right), i=1, \ldots, p+1, \omega_{i} \in[0,1], x=\left(x_{1}, x_{2}, \ldots, x_{p+1}\right)$ and $\omega=\left(\omega_{1}\right.$, $\left.\ldots, \omega_{p+1}\right) \in[0,1]^{p+1} \equiv p^{p+1}$. As usual, it is convenient to write $S_{T}(x)$ and $S(x)$ in terms of $F_{1}^{-1}($.$) . Let us define F_{T j}(\xi)=T^{-1} \sum_{t=1}^{T} 1\left\{X_{t+j-1} \leqslant F_{1}^{-1}(\xi)\right\}$, for $\xi \in[0,1]$. Then,

$$
S_{T}(x)=S_{T}(\omega)=T^{-1} \sum_{t=1}^{T} \prod_{j=1}^{p+1} 1\left\{X_{t+j-1} \leqslant F_{1}^{-1}\left(\omega_{j}\right)\right\}-\prod_{j=1}^{p+1} F_{T j}\left(\omega_{j}\right)
$$

and

$$
S(x)=S(\omega)=E\left[\prod_{j=1}^{p+1} 1\left\{X_{j} \leqslant F_{1}^{-1}\left(\omega_{j}\right)\right\}\right]-\prod_{j=1}^{p+1} \omega_{j} .
$$

The following lemma is useful for deriving the asymptotic distribution of $S_{t}(\omega)$ under $\mathrm{H}_{0}$.

Lemma 1. Under $\mathrm{H}_{0}$,

$$
\sup _{\omega \in I^{p+1}}\left|S_{T}(\omega)-T^{-1} \sum_{t=1}^{T} R_{t}(\omega)\right|=\mathrm{O}\left(T^{-1}\right) \text { a.s. }
$$

where

$$
\begin{aligned}
R_{t}(\omega)= & \prod_{j=1}^{p+1} 1\left\{X_{t+j-1} \leqslant F_{1}^{-1}\left(\omega_{j}\right)\right\}-\sum_{j=1}^{p+1} 1\left\{X_{t+j-1} \leqslant F_{1}^{-1}\left(\omega_{j}\right)\right\} \prod_{i=1, i \neq j}^{p+1} \omega_{i} \\
& +p \prod_{j=1}^{p+1} \omega_{j} .
\end{aligned}
$$

Let us define $\omega^{\prime}=\left(\omega_{1}^{\prime}, \ldots, \quad \omega_{p+1}^{\prime}\right) \in P^{p+1}$ and $\omega_{i}^{\prime}=F_{\mathrm{I}}\left(x_{i}^{\prime}\right)$. Routine calculations show that, under $\mathrm{H}_{0}$,

$$
E\left\{R_{t}(\omega)\right\}=0, \quad E\left\{R_{t}(\omega) R_{s}\left(\omega^{\prime}\right)\right\}=0, \quad \text { all } t, s \text { such that }|s-t| \geqslant p .
$$

Then, under $\mathrm{H}_{0}$ 


$$
\begin{aligned}
\Xi\left(\omega, \omega^{\prime}\right) & =T^{-1} \sum_{t=1}^{T} \sum_{s=1}^{T} E\left\{R_{t}(\omega) R_{s}\left(\omega^{\prime}\right)\right\} \\
& =\sum_{s=1-p}^{p-1} E\left\{R_{p+s}(\omega) R_{p}\left(\omega^{\prime}\right)\right\} \\
& =\sum_{s=1-p}^{p-1} \gamma_{s}\left(\omega, \omega^{\prime}\right)
\end{aligned}
$$

where, for $s=0,1, \ldots, p-1, p \geqslant 1, \gamma_{s}\left(\omega, \omega^{\prime}\right)=\gamma_{-s}\left(\omega^{\prime}, \omega\right)$,

$$
\gamma_{0}\left(\omega, \omega^{\prime}\right)=\prod_{j=1}^{p+1} \min \left(\omega_{j}, \omega_{j}^{\prime}\right)-\sum_{j=1}^{p+1} \min \left(\omega_{j}, \omega_{j}^{\prime}\right) \prod_{i=1, i \neq j}^{p+1} \omega_{i} \omega_{i}^{\prime}+p \prod_{j=1}^{p+1} \omega_{j} \omega_{j}^{\prime}
$$

and for $l=1,2, \ldots, p-1, p \geqslant 2$,

$$
\begin{aligned}
\gamma_{l}\left(\omega, \omega^{\prime}\right)= & \prod_{j=1}^{p+1-l} \min \left(\omega_{j}, \omega_{j+l}^{\prime}\right) \prod_{j=1}^{l} \omega_{j}^{\prime} \prod_{j=p+2-l}^{p+1} \omega_{j} \\
& -\sum_{j=1}^{p+1-l} \min \left(\omega_{j}, \omega_{j+l}^{\prime}\right) \prod_{i=1, i \neq j}^{p+1} \omega_{i} \prod_{i=1, i \neq j+l}^{p+1} \omega_{i}^{\prime} \\
& +(p-l) \prod_{j=1}^{p+1} \omega_{j} \omega_{j}^{\prime} .
\end{aligned}
$$

Thus, $T^{1 / 2} S_{T}(\omega)$ has zero asymptotic mean and the asymptotic covariance between $T^{\mathrm{l} / 2} S_{T}(\omega)$ and $T^{1 / 2} S_{T}\left(\omega^{\prime}\right)$ is $\Xi\left(\omega, \omega^{\prime}\right)$.

Let us introduce the $(p+1)$-dimensional Brownian bridge $W^{0}(\omega), \omega=\left(\omega_{1}\right.$, $\left.\ldots, \omega_{p+1}\right) \in I^{p+1} \equiv[0,1]^{p+1}$; i.e.

$$
W^{0}(\omega)=W(\omega)-W(1,1, \ldots, 1) \prod_{j=1}^{p+1} \omega_{j}
$$

where $W($.$) is the standard Wiener random field on p^{p+1}$. The process

$$
W^{\dagger}(\omega)=W^{0}(\omega)-\sum_{i=1}^{p+1} W^{0}\left(\omega_{(i)}\right) \prod_{j=1, j \neq i}^{p+1} \omega_{j}
$$

where $\omega_{(i)}=\left(1,1, \ldots, 1, \omega_{i}, 1, \ldots, 1\right)$ is a vector of ones with $\omega_{i}$ in the $i$ th place, is a separable Gaussian process with $E\left\{W^{\dagger}(\omega)\right\}=0$, and for $\omega^{\prime}=\left(\omega_{1}^{\prime}, \ldots\right.$, $\left.\omega_{p+1}^{\prime}\right), E\left\{W^{\dagger}(\omega) W^{\dagger}\left(\omega^{\prime}\right)\right\}=\gamma_{0}\left(\omega, \omega^{\prime}\right)$. That is, when $p=1$, the empirical process $T^{1 / 2} S_{T}(\omega)$ has the same asymptotic mean and covariances as $W^{\dagger}(\omega)$. Furthermore, Skaug and Tjøstheim (1993) have proved that

$$
B_{T} \stackrel{d}{\rightarrow} B \equiv \int_{I^{2}} W^{\dagger}(\omega)^{2} d \omega, \quad \text { when } p=1
$$


Then, in the case $p=1, B_{T}$ has the same asymptotic distribution as the HBKR statistic for testing independence of two random variables. In this case, Blum et al. (1961) have found the characteristic function and have tabulated the corresponding distribution.

When $p \geqslant 2$, the asymptotic covariances of $T^{1 / 2} S_{T}(\omega)$ differ from those of $W^{\dagger}(\omega)$. Let $W^{*}(\omega), \omega \in I^{p+1}$, be a separable Gaussian process with $E\left\{W^{*}(\omega)\right\}=0$, and for $\omega^{\prime} \in P^{p+1}, E\left\{W^{*}(\omega) W^{*}\left(\omega^{\prime}\right)\right\}=\Xi\left(\omega, \omega^{\prime}\right)$. The following theorem, proved in the Appendix, states the asymptotic null distribution of $B_{T}$ in the general case.

THEOREM 1. Under $\mathrm{H}_{0}, B_{T} \stackrel{d}{\rightarrow} B \equiv \int_{p^{p+1}} W^{*}(\omega)^{2} d \omega$, for any $p \geqslant 1$.

Let us define $B_{\alpha}$ as $\operatorname{Pr}\left(B>B_{\alpha}\right)=\alpha$. Whenever $B_{\alpha}$ is available, an asymptotic test at the $\alpha$ level of significance consists of rejecting $\mathrm{H}_{0}$ when the observed value of $B_{T}$, for a given sample, is greater than $B_{\alpha x}$.

The consistency of the test follows whenever a Glivenko-Cantelli theorem under the alternative hypothesis is provided. The following theorem, proved in the Appendix, states that the test is consistent when the sequence of observations is ergodic.

THEOREM 2. Under $\mathrm{H}_{1}$, assuming that $\left\{X_{t}, t \geqslant 1\right\}$ is an ergodic sequence, then

$$
\lim _{n \rightarrow \infty} \operatorname{Pr}\left(B_{T}>B_{\alpha}\right)=1 \text {. }
$$

Calculation of the critical values $B_{a}$ does not seem an easy task. Cotterill and Csörgö (1985) have found the characteristic function of $\int_{p^{p+1}} W^{\dagger}(\omega)^{2} d \omega$, and have tabulated its distribution, for $p>1$. The permutation test discussed in the next section is easy to implement and enjoys good level and power properties.

\section{COMPUTING CRITICAL VALUES BY RANDOM PERMUTATION}

An exact test based on the statistic $B_{T}$ consists of rejecting $\mathrm{H}_{0}$ when the observed statistic value exceeds $B_{T \alpha}$ defined as

$$
\operatorname{Pr}\left(B_{T} \leqslant B_{T \alpha} \mid H_{0}\right)=1-\alpha .
$$

The main practical difficulty with hypotheses tests comes in calculating $B_{T \alpha}$. Exact critical values are usually approximated based on the asymptotic distribution of the statistics. Alternatively, we can perform an exact permutation test. These tests were first proposed by Fisher (1935), and they are discussed in the books by Edgington (1987), Noreen (1989) and Efron and Tibshirani (1993). In a time series context, they have been applied by Chan and Tran (1992). Exact critical values can also be approximated by bootstrap. Romano (1989) provides some arguments in favour of permutation tests. He shows that the difference 
between the critical functions of permutation and bootstrap tests evaluated at the observed data converges to zero in probability. So, permutation tests may be preferable since an exact desired level of the test can be obtained for finite samples.

Let $\left\{\eta_{1}, \ldots, \eta_{T+p}\right\}$ be a random permutation of the integers $\{1, \ldots, T+p\}$. So, we construct the sample $X^{*}=\left\{Z_{1}^{*}, \ldots, Z_{T}^{*}\right\}$, where $Z_{t}^{*}=\left\{X_{\eta_{t}}, \ldots, X_{\eta_{t+p}}\right\}$, and from this sample we calculate the statistic $B_{T}^{*}$, constructed in the same way as $B_{T}$ but employing the sample $X^{*}$.

Under $\mathrm{H}_{0}$ and $\mathrm{H}_{1}, B_{T}^{*} \stackrel{d}{\rightarrow} B$ as $T \rightarrow \infty$. At each random permutation, for $T$ fixed, we are equally likely to obtain any one of the possible samples $X_{i}^{+}=$ $\left\{Z_{1 i}^{+}, \ldots, Z_{T i}^{+}\right\}$, where $Z_{t i}^{+}=\left\{X_{\xi_{t}^{i}}, \ldots, X_{\xi_{t+p}^{i}}\right\}$ and $\left\{\xi_{t}^{i}, \ldots, \xi_{t+p}^{i}\right\}, i=1, \ldots$, $(T+p)$ !, are all different permutations with integers $(1,2, \ldots, T+p)$. Thus, under $\mathrm{H}_{0}$ and $\mathrm{H}_{1}, B_{T}^{*}$ has probability $1 /(T+p)$ ! of equalling any one of its possible values $B_{i T}^{+}, i=1, \ldots,(T+p)$ !, where $B_{i T}^{+}$is constructed in the same way as $B_{T}$ but with the sample $X_{i}^{+}$. However, under the alternative hypothesis, some values are more likely than others. The permutation test consists of rejecting the null hypothesis when $B_{T}$ exceeds the critical value $B_{T \alpha}^{*}$, defined as

$$
\{(T+p) !\}^{-1} \sum_{i=1}^{(T+p) !} 1\left(B_{i T}^{+}>B_{T \alpha}^{*}\right)=1-\alpha .
$$

Like other permutation tests, not all significance levels are possible. That is, $B_{T a}^{*}$ is not necessarily unique. The test can also be based on the $p$-values computed as $\{(T+p) !\}^{-1} \sum_{i=1}^{(T+p) !} 1\left(B_{i T}^{+}>B_{T}\right)$.

Calculation of the critical values $B_{T \alpha}^{*}$ is computationally demanding, but they can be approximated by repeated resampling, using random permutation. That is, $M$ random permutations of the integers $(1, \ldots, T+p),\left\{\eta_{t}^{i}, \ldots, \eta_{t+p}^{i}\right\}, i=1$, $\ldots, M$, are drawn and, for each permutation, we compute $B_{i T}^{*}, i=1, \ldots, M$, in the same way as $B_{T}$ but using samples $X_{i}^{*}=\left\{Z_{1 i}^{*}, \ldots, Z_{T i}^{*}\right\}$, and $Z_{t i}^{*}=\left\{X_{\eta^{i}}\right.$, $\left.\ldots, X_{\eta_{i+p}}\right\}$. In fact, we are sampling with replacement from all possible $(T+p)$ ! samples $X_{i}^{+}=\left\{Z_{1 i}^{+}, \ldots, Z_{T i}^{+}\right\}$. Then $B_{T \alpha}^{*}$ is approximated as accurately as desired by $B_{T \alpha}^{(M)}$, where

$$
M^{-1} \sum_{j=1}^{M} 1\left(B_{j T}^{*} \leqslant B_{T \alpha}^{(M)}\right)=1-\alpha .
$$

Similarly, the $p$-value is approximated by $M^{-1} \sum_{j=1}^{M} 1\left(B_{j T}^{*}>B_{T}\right)$.

The asymptotic test based on critical values $B_{\alpha}$ and the permutation test based on $B_{T \alpha}^{*}$ are asymptotically equivalent. The following Monte Carlo experiment illustrates the performance of both tests with small samples.

\section{MONTE CARLO EXPERIMENTS AND AN EMPIRICAL EXAMPLE USING EXCHANGE RATE DATA}

In these Monte Carlo experiments we compare the empirical power function of the test based on the statistic $B_{T}$ and the popular Ljung-Box $Q$ statistic (Ljung 
and Box, 1978), based on the squared correlation coefficient. Both test are only computed for $p=1$.

The empirical power function is computed as the proportion of times that $\mathrm{H}_{0}$ is rejected in 5000 replications of the time series model. We consider tests where the critical values are approximated from the quantiles of the asymptotic null distribution of the corresponding statistic, and from the random permutation procedure presented in Section 3. We only consider first-order dependence alternatives $(p=1)$ in the following two models,

$$
\begin{aligned}
X_{t} & =b X_{t-1}+\varepsilon_{t} & \text { where } \varepsilon_{t} & \sim \text { i.i.d. } \mathrm{N}(0,1) \\
X_{t} & =b \varepsilon_{t-1}^{2}+\varepsilon_{t} & \text { where } \varepsilon_{t} & \sim \text { i.i.d. } \mathrm{N}(0,1)
\end{aligned}
$$

The $Q$ test is more powerful than any other when the dependence structure is linear. Then, the $Q$ test is expected to work better than the empirical distribution function (EDF) test in the AR(1) model (5.1), while the EDF test is expected to perform better in the nonlinear MA(1) model (5.2).

The empirical power function has been computed for $T=20,50,100$ and different values of the parameter $b$ in the two models. The critical values in the random permutation test are computed using 500 random permutations. The results for model (5.1) are in Table I and the results for model (5.2) are in Table II. Both tables indicate that the random permutation test enjoys good level, even for the smallest sample size, and the power is not worse than the test based on asymptotic critical values. The resampled procedure is time consuming, since at each replication the generated sample has to be permuted 500 times. Of course, level accuracy is expected to increase as the number of random permutations increases.

In model (5.1) the EDF and $Q$ tests behave similarly, both in their asymptotic and permutation test versions, for any value of $b$ and any significance level $\alpha$. However, the EDF test overperforms the $Q$ test in model (5.2), which exhibits a more subtle dependence structure.

Table III summarizes an empirical application of the test, in the asymptotic and resampled versions, to testing serial independence of exchange rate changes using New York stock market data. The observations are $X_{t}=\log P_{t}-\log P_{t-\mathrm{b}}$ where $P_{t}$ is the exchange rate in period $t$. The data are recorded, from January 1977 to April 1988, daily, monthly and quarterly for three currencies against the US dollar: pound sterling, deutschmark and Japanese yen.

The effectiveness of several serial independence tests has been tried with this sort of data. Whistler (1990) found, using UK data recorded in a similar period, that based on parametric autoregressive conditional heteroskedasticity (ARCH) models, the independence hypothesis is rejected employing daily data but it cannot be rejected with monthly data. Based on the same data, Robinson (1991) rejected the independence null hypothesis in all cases, using his entropybased nonparametric test. With the same data, Pinkse (1993) also rejected the null hypothesis in all cases using a nonparametric test based on the empirical characteristic function.

With daily observations, our test always rejects the null hypothesis. However, 
TABLE I

Empirical Power Function of $B_{T}$ Based on 5000 Replications of THe MOdel $x_{t}=b x_{t-1}+\varepsilon_{t}, t=1, \ldots, T$, Where $\varepsilon_{t} \sim$ i.i.d. N $(0,1)$, for $T=20,50,100$

\begin{tabular}{|c|c|c|c|c|c|c|c|c|c|c|c|c|}
\hline & \multicolumn{3}{|c|}{$\begin{array}{c}\mathrm{EDF} \\
\text { permutation test }\end{array}$} & \multicolumn{3}{|c|}{$\begin{array}{c}\text { EDF } \\
\text { asymptotic test }\end{array}$} & \multicolumn{3}{|c|}{$\frac{Q}{\text { permutation test }}$} & \multicolumn{3}{|c|}{$\frac{Q}{\text { asymptotic test }}$} \\
\hline & $T=20$ & $T=50$ & $T=100$ & $T=20$ & $T=50$ & $T=100$ & $T=20$ & $T=50$ & $T=100$ & $T=20$ & $T=50$ & $T=100$ \\
\hline \multicolumn{13}{|l|}{$\alpha=0.05$} \\
\hline$b=0$ & 0.0566 & 0.0518 & $0.05 \mathrm{I} 2$ & 0.0626 & 0.0532 & 0.0512 & 0.0520 & 0.0528 & 0.0524 & 0.0668 & 0.0558 & 0.0508 \\
\hline$b=0.1$ & 0.0786 & 0.1192 & 0.1555 & 0.0860 & 0.1224 & 0.1524 & 0.0502 & 0.0872 & 0.1320 & 0.0610 & 0.0902 & 0.1344 \\
\hline$b=0.2$ & 0.1424 & 0.2554 & 0.4526 & 0.1592 & 0.2628 & 0.4562 & 0.0798 & 0.2264 & 0.4550 & 0.0982 & 0.2330 & 0.4552 \\
\hline$b=0.3$ & 0.2230 & 0.4902 & 0.7928 & 0.2400 & 0.4940 & 0.7940 & 0.1536 & 0.4810 & 0.8204 & 0.1874 & 0.4896 & 0.8214 \\
\hline$b=0.4$ & 0.3479 & 0.7300 & 0.9540 & 0.3708 & 0.7370 & 0.9544 & 0.2676 & 0.7364 & 0.9686 & 0.3044 & 0.7512 & 0.9678 \\
\hline$b=0.5$ & 0.4930 & 0.8978 & 0.9948 & 0.5136 & 0.9004 & 0.9950 & 0.4204 & 0.9056 & 0.9966 & 0.4676 & 0.9126 & 0.9972 \\
\hline$b=0.6$ & 0.6216 & 0.9686 & 0.9996 & 0.6380 & 0.9698 & 0.9996 & 0.5760 & 0.9776 & 1.0000 & 0.6156 & 0.9802 & 1.0000 \\
\hline$b=0.7$ & 0.7470 & 0.9920 & 1.0000 & 0.7658 & 0.9922 & 1.0000 & 0.7200 & 0.9950 & 1.0000 & 0.7518 & 0.9945 & 1.0000 \\
\hline$b=0.8$ & 0.8292 & 0.9976 & 1.0000 & 0.8416 & 0.9982 & 1.0000 & 0.8106 & 0.9998 & 1.0000 & 0.8320 & 0.9998 & 1.0000 \\
\hline$b=0.9$ & 0.8950 & 0.9998 & 1.000 & 0.9042 & 0.9998 & 1.0000 & 0.8862 & 0.9994 & 1.0000 & 0.9034 & 0.9994 & 1.0000 \\
\hline \multicolumn{13}{|l|}{$\alpha=0.01$} \\
\hline$b=0$ & 0.0128 & 0.0128 & 0.0122 & 0.0112 & 0.0112 & 0.0090 & 0.0122 & 0.0126 & 0.0110 & 0.0124 & 0.0114 & 0.0092 \\
\hline$b=0.1$ & 0.0246 & 0.0459 & 0.0570 & 0.0220 & 0.0388 & 0.0494 & 0.0124 & 0.0248 & 0.0456 & 0.0120 & 0.0226 & 0.0393 \\
\hline$b=0.2$ & 0.0476 & 0.1166 & 0.2542 & 0.0466 & 0.1094 & 0.2392 & 0.0232 & 0.0890 & 0.2596 & 0.0244 & 0.0830 & 0.2368 \\
\hline$b=0.3$ & 0.0938 & 0.2932 & 0.5946 & 0.0902 & 0.2796 & 0.5754 & 0.0488 & 0.2780 & 0.6298 & 0.0502 & 0.2596 & 0.6192 \\
\hline$b=0.4$ & 0.1744 & 0.5212 & 0.8716 & 0.1740 & 0.5140 & 0.8670 & 0.1186 & 0.5330 & 0.9034 & 0.1228 & 0.5250 & 0.8986 \\
\hline$b=0.5$ & 0.2834 & 0.7592 & 0.9796 & 0.2880 & 0.7512 & 0.9788 & 0.2164 & 0.7836 & 0.9890 & 0.2252 & 0.7804 & 0.9886 \\
\hline$b=0.6$ & 0.4264 & 0.9074 & 0.9984 & 0.4260 & 0.9114 & 0.9984 & 0.3688 & 0.9320 & 0.9996 & 0.3714 & 0.9288 & 0.9998 \\
\hline$b=0.7$ & 0.5646 & 0.9762 & 0.9998 & 0.5704 & 0.9744 & 1.0000 & 0.5298 & 0.9836 & 1.0000 & 0.5402 & 0.9838 & 1.0000 \\
\hline$b=0.8$ & 0.6969 & 0.9932 & 1.0000 & 0.7022 & 0.9936 & 1.0000 & 0.6566 & 0.9956 & 1.0000 & 0.6694 & 0.9948 & 1.0000 \\
\hline$b=0.9$ & 0.7990 & 0.9984 & 1.0000 & 0.7998 & 0.9980 & 1.0000 & 0.7846 & 0.9988 & 1.0000 & 0.7876 & 0.9986 & 1.0000 \\
\hline
\end{tabular}


TABLE II

EMPIRICAL POWER FUnCtion OF $B_{T}$ BASEd ON 5000 RePlications of THE MODEl $x_{t}=b \varepsilon_{t-1}^{2}+\varepsilon_{t}, t=1, \ldots, T$, where $\varepsilon_{t} \sim$ i.i.d. N $(0,1)$, FOR $T=20,50,100$

\begin{tabular}{|c|c|c|c|c|c|c|c|c|c|c|c|c|}
\hline & \multicolumn{3}{|c|}{$\begin{array}{c}\mathrm{EDF} \\
\text { permutation test }\end{array}$} & \multicolumn{3}{|c|}{$\begin{array}{c}\text { EDF } \\
\text { asymptotic test }\end{array}$} & \multicolumn{3}{|c|}{$\frac{Q}{\text { permutation test }}$} & \multicolumn{3}{|c|}{$\frac{Q}{\text { asymptotic test }}$} \\
\hline & $T=20$ & $T=50$ & $T=100$ & $T=20$ & $T=50$ & $T=100$ & $T=20$ & $T=50$ & $T=100$ & $T=20$ & $T=50$ & $T=100$ \\
\hline \multicolumn{13}{|l|}{$\alpha=0.05$} \\
\hline & 0.0566 & 0.0518 & 0.0512 & 0.0626 & 0.0532 & 0.0512 & 0.0552 & 0.0528 & 0.0524 & 0.0668 & 0.0558 & 0.0508 \\
\hline$b=0.1$ & 0.0500 & 0.0608 & 0.0578 & 0.0568 & 0.0620 & 0.0570 & 0.0544 & 0.0622 & 0.0596 & 0.0692 & 0.0660 & 0.0584 \\
\hline$b=0.2$ & 0.0554 & 0.0680 & 0.0954 & 0.0652 & 0.0698 & 0.0936 & 0.0660 & 0.0786 & 0.0822 & 0.0816 & 0.0822 & 0.0814 \\
\hline$b=0.3$ & 0.0602 & 0.0938 & 0.1736 & 0.0698 & 0.0958 & 0.1740 & 0.0844 & 0.0944 & 0.1056 & 0.1014 & 0.0974 & 0.1040 \\
\hline$b=0.4$ & 0.0728 & 0.1250 & 0.2472 & 0.0786 & 0.1290 & 0.2508 & 0.0982 & 0.1190 & 0.1288 & 0.1150 & 0.1222 & 0.1298 \\
\hline$b=0.5$ & 0.0820 & 0.1388 & 0.3326 & 0.0936 & 0.1378 & 0.3292 & 0.1020 & 0.1362 & 0.1388 & 0.1226 & 0.1406 & 0.1382 \\
\hline$b=0.6$ & 0.0806 & 0.1474 & 0.3772 & 0.0910 & 0.1508 & 0.3740 & 0.1116 & 0.1356 & 0.1460 & 0.1230 & 0.1364 & 0.1414 \\
\hline$b=0.7$ & 0.0926 & 0.1698 & 0.4052 & 0.1030 & 0.1762 & 0.4084 & 0.1202 & 0.1526 & 0.1600 & 0.1286 & 0.1490 & 0.1572 \\
\hline$b=0.8$ & 0.0872 & 0.1672 & 0.4414 & 0.0954 & 0.1726 & 0.4366 & 0.1078 & 0.1488 & 0.1654 & 0.1142 & 0.1424 & 0.1552 \\
\hline$b=0.9$ & 0.1008 & 0.1740 & 0.4410 & 0.1074 & 0.1780 & 0.4370 & 0.1290 & 0.1444 & 0.1556 & 0.1228 & 0.1368 & 0.1476 \\
\hline \multicolumn{13}{|l|}{$\alpha=0.01$} \\
\hline$b=0$ & 0.0128 & 0.0128 & 0.0122 & 0.0112 & 0.0112 & 0.0090 & 0.0122 & 0.0126 & 0.0110 & 0.0124 & 0.0114 & 0.0092 \\
\hline$b=0.1$ & 0.0152 & 0.0160 & 0.0120 & 0.0138 & 0.0142 & 0.0102 & 0.0122 & 0.0174 & 0.0134 & 0.0116 & 0.0152 & 0.0114 \\
\hline$b=0.2$ & 0.0110 & 0.0174 & 0.0246 & 0.0108 & 0.0156 & 0.0206 & 0.0166 & 0.0218 & 0.0232 & 0.0164 & 0.0198 & 0.0188 \\
\hline$b=0.3$ & 0.0166 & 0.0236 & 0.0462 & 0.0172 & 0.0196 & 0.0400 & 0.0226 & 0.0352 & 0.0366 & 0.0250 & 0.0360 & 0.0320 \\
\hline$b=0.4$ & 0.0182 & 0.0348 & 0.0680 & 0.0182 & 0.0290 & 0.0552 & 0.0286 & 0.0416 & 0.0498 & 0.0280 & 0.0359 & 0.0452 \\
\hline$b=0.5$ & 0.0234 & 0.0400 & 0.0960 & 0.0226 & 0.0378 & 0.0782 & 0.0298 & 0.0556 & 0.0574 & 0.0272 & 0.0448 & 0.0494 \\
\hline$b=0.6$ & 0.0262 & 0.0434 & 0.1174 & 0.0254 & 0.0388 & 0.1008 & 0.0370 & 0.0530 & 0.0588 & 0.0300 & 0.0436 & 0.0512 \\
\hline$b=0.7$ & 0.0276 & 0.0522 & 0.1352 & 0.0280 & 0.0488 & 0.1148 & 0.0454 & 0.0538 & 0.0710 & 0.0372 & 0.0434 & 0.0590 \\
\hline$b=0.8$ & 0.0262 & 0.0508 & 0.1490 & 0.0268 & 0.0450 & 0.1280 & 0.0392 & 0.0584 & 0.0664 & 0.0320 & 0.0446 & 0.0538 \\
\hline$b=0.9$ & 0.0270 & 0.0542 & 0.1555 & 0.0272 & 0.0476 & 0.1342 & 0.0384 & 0.0570 & 0.0680 & 0.0298 & 0.0428 & 0.0560 \\
\hline
\end{tabular}


TABLE III

Statistic Values, Resampled Critical Values and $p$ Values from Exchange Rate Change Data of Pound Sterling, Yen and Deutschmark with Respect to the US Dollar in the New York Stock Market from January 1977 to APRIL 1988 (2832 Daily, 158 MonThly And 53 QUARTERLY OBSERVATIONS)

\begin{tabular}{|c|c|c|c|c|c|c|}
\hline & \multicolumn{2}{|c|}{ Daily } & \multicolumn{2}{|c|}{ Monthly } & \multicolumn{2}{|c|}{ Quarterly } \\
\hline & EDF & $Q$ & EDF & $Q$ & $\mathrm{EDF}$ & $Q$ \\
\hline \multicolumn{7}{|c|}{ Sterling pound/US dollar } \\
\hline Statistic & 0.140 & 0.076 & 0.071 & 0.416 & 0.016 & 0.001 \\
\hline \multicolumn{7}{|l|}{ Critical values } \\
\hline \multicolumn{7}{|l|}{$\alpha=0.05$} \\
\hline Asymptotic & 0.058 & 3.840 & 0.058 & 3.840 & 0.058 & 3.840 \\
\hline Resampled & 0.060 & 3.836 & 0.055 & 3.468 & 0.060 & 4.211 \\
\hline \multicolumn{7}{|l|}{$\alpha=0.01$} \\
\hline Asymptotic & 0.087 & 6.630 & 0.087 & 6.630 & 0.087 & 6.630 \\
\hline Resampled & 0.093 & 6.171 & 0.079 & 5.544 & 0.087 & 6.406 \\
\hline$p$ value (resampled) & 0.002 & 0.796 & 0.015 & 0.507 & 0.771 & 0.986 \\
\hline \multicolumn{7}{|c|}{ Deutschmark/US dollar } \\
\hline Statistic & 0.158 & 7.326 & 0.071 & 0.170 & 0.017 & 0.053 \\
\hline \multicolumn{7}{|l|}{ Critical values } \\
\hline \multicolumn{7}{|l|}{$\alpha=0.05$} \\
\hline Asymptotic & 0.058 & 3.840 & 0.058 & 3.840 & 0.058 & 3.840 \\
\hline Resampled & 0.059 & 3.582 & 0.057 & 3.758 & 0.057 & 3.713 \\
\hline \multicolumn{7}{|l|}{$\alpha=0.01$} \\
\hline Asymptotic & 0.087 & 6.630 & 0.087 & 6.630 & 0.087 & 6.630 \\
\hline Resampled & 0.082 & 5.645 & 0.085 & 6.664 & 0.084 & 6.427 \\
\hline$p$ value (resampled) & 0.000 & 0.004 & 0.0269 & 0.880 & 0.712 & 0.842 \\
\hline \multicolumn{7}{|l|}{ Yen/US dollar } \\
\hline Statistic & 0.112 & 2.389 & 0.081 & 0.978 & 0.083 & 2.447 \\
\hline \multicolumn{7}{|l|}{ Critical values } \\
\hline \multicolumn{7}{|l|}{$\alpha=0.05$} \\
\hline Asymptotic & 0.058 & 3.840 & 0.058 & 3.840 & 0.058 & 3.840 \\
\hline Resampled & 0.061 & 3.990 & 0.054 & 3.664 & 0.057 & 3.539 \\
\hline \multicolumn{7}{|l|}{$\alpha=0.01$} \\
\hline Asymptotic & 0.087 & 6.630 & 0.087 & 6.630 & 0.087 & 6.630 \\
\hline Resampled & 0.100 & 6.230 & 0.073 & 5.673 & 0.081 & 5.691 \\
\hline$p$ value (resampled) & 0.003 & 0.115 & 0.007 & 0.338 & 0.005 & 0.105 \\
\hline
\end{tabular}

Note: Resampled critical values are computed using 1000 random permutations of the original series.

the $Q$ test only detects linear dependence in the deutschmark case. Using monthly observations, our statistic takes values above the asymptotic and permutation test critical values at the $\alpha=0.05$ level of significance in all cases. However, at the $\alpha=0.01$ level, the null hypothesis is not rejected by our test in any of its versions for all currencies except the yen. In the yen case, the observed value of the statistic is greater than the resampled critical value. This is not a surprise. Plotting the squared series, a structural change is clearly observed. There is more volatility in the first part of the sample. This may induce rejection of the null hypothesis. This argument also explains why, using quarterly data, the independence hypothesis cannot be rejected, with all tests, 
for the pound and deutschmark, but it is rejected for the yen. Using quarterly data for the yen, our test rejects clearly the null hypothesis, and the resampled $p$ value of the $Q$ test is only 0.105 .

\section{CONCLUSIONS}

In this paper we have proposed a test of serial independence against serial dependence of fixed and finite order. The test is based on the property that under independence the joint distribution function of a set of random variables is equal to the product of the marginal distribution functions. The test statistics compare the joint and marginal sample distribution functions in the same way as Hoeffding (1948) and Blum et al. (1961). This sort of statistic has also been used by Skaug and Tjøstheim (1993) for testing pairwise independence of the elements in $\left(X_{t}, X_{t+1}, \ldots, X_{t+p}\right)$ by means of a combination of first-order serial independence tests. In this paper we test complete independence of the whole set $\left(X_{t}, X_{t+1}, \ldots, X_{t+p}\right)$ using the original formulation of Blum et al. (1961). We find that when $p=1$ the test statistic has the same null distribution as the HBKR test statistic. However, for $p>1$ the asymptotic null distribution differs from that of Blum et al. (1961) in a nonobvious way. We also propose to approximate the critical values by an approximate permutation test. Monte Carlo simulations and a small application to testing the random walk hypothesis of exchange rates of return for different currencies, illustrates the good performance of the test in small samples.

Our test is consistent in the direction of broader alternatives than existing tests. However, in practice, other tests designed for specific alternative hypotheses are possibly more powerful. It may be interesting to study the finite sample properties of available serial independence tests under different alternatives by means of simulations.

The testing procedure is interesting for specification testing of parametric time series models applying the statistic to the residuals. The study of the asymptotic properties of our test under such circumstances is left for further work.

\section{APPENDIX}

ProOF OF Lemma 1. It suffices to prove that for all $k \geqslant 2, k$ fixed,

$$
\sup _{\omega^{k} \in I^{k}}\left|D_{k}\left(\omega^{k}\right)\right|=\mathrm{O}\left(T^{-1}\right) \text { a.s. }
$$

where $\omega^{k}=\left(\omega_{1}, \omega_{2}, \ldots, \omega_{k}\right)^{\prime}$ and

$$
D_{k}\left(\omega^{k}\right)=\prod_{j=1}^{k} F_{T j}\left(\omega_{j}\right)-\prod_{j=1}^{k} \omega_{j}-\sum_{j=1}^{k}\left\{F_{T j}\left(\omega_{j}\right)-\omega_{j}\right\} \prod_{\substack{i, 1 \\ i, j}}^{k} \omega_{i} .
$$


We prove (A.1) by induction using

$$
\sup _{\xi \in[0,1]}\left|F_{T j}(\xi)-\xi\right|=\mathrm{O}\left(T^{-1 / 2}\right) \text { a.s. }
$$

see, for example, Brillinger (1969). For $k=2$,

$$
D_{2}\left(\omega^{2}\right)=\left\{F_{T 1}\left(\omega_{1}\right)-\omega_{1}\right\}\left\{F_{T 2}\left(\omega_{2}\right)-\omega_{2}\right\}
$$

and (A.1) with $k=2$ follows using (A.2). Assume (A.1) holds for $k=p$. Then for $k=p+1$, notice that

$$
\begin{aligned}
D_{p+1}\left(\omega^{p+1}\right)= & \omega_{p+1} D_{p}\left(\omega^{p}\right)+\left\{F_{T p+1}\left(\omega_{p+1}\right)-\omega_{p+1}\right\} D_{p}\left(\omega^{p}\right) \\
& +\left\{F_{T p+1}\left(\omega_{p+1}\right)-\omega_{p+1}\right\} \sum_{j=1}^{p}\left\{F_{T j}\left(\omega_{j}\right)-\omega_{j}\right\} \prod_{\substack{i=1 \\
i \neq j}}^{p} \omega_{i}
\end{aligned}
$$

and, since (A.1) holds for $k=p$, (A.1) follows with $k=p+1$ using (A.2).

Proof OF THEOREM 1. In order to obtain the infinite dimensional asymptotic distribution of $T^{1 / 2} S_{T}(\omega)$ in the general case, we first note that

$$
T^{-1} \sum_{t=1}^{T} R_{t}(\omega)=(p+1)^{-1} \sum_{j=0}^{p} S_{j N}(\omega)
$$

where $R_{t}($.$) was defined in Lemma 1$ and

$$
S_{j N}(\omega)=N^{-1} \sum_{i=1}^{N} R_{p t+t-j}(\omega), \quad j=0, \ldots, p
$$

$N=T /(p+1)$, and assuming, without lost of generality, that $N$ is an integer. Under $\mathrm{H}_{0}$, for all $j=0, \ldots, p$, the random vectors $\left\{Z_{p t+t-j}, t=1, \ldots, N\right\}$ are serially independent remember that $Z_{t}=\left(X_{t}, X_{t+1}, \ldots, X_{t+p}\right)-$ and also the $p+1$ components of the vector $Z_{p t+t-j}, t \geqslant 1$, are completely independent. Then, using Blum et al.'s (1961) results, the infinite dimensional asymptotic distribution of each $S_{j N}(\omega)$ is that of $W_{j}^{\dagger}(\omega), j=0, \ldots, p$, where each $W_{j}^{\dagger}(\omega)$ is distributed as $W^{\dagger}(\omega)$. Furthermore, using Theorem 3 in Csörgö (1979),

$$
\sup _{\omega \in p^{p+1}}\left|N^{1 / 2} S_{j N}(\omega)-W_{j N}^{\dagger}(\omega)\right|=\mathrm{O}\left\{N^{-1 / 2(p+2)}(\log N)^{3 / 2}\right\} \text { a.s. } \quad p \geqslant 1, j=0, \ldots, p
$$

and

$$
\sup _{\omega \in I^{p+1}}\left|N S_{j N}(\omega)\right|=\mathbf{O}\left\{(N \log \log N)^{1 / 2}\right\} \text { a.s. } \quad p \geqslant 1, j=0, \ldots, p
$$

where each $\left\{W_{j N}^{\dagger}(\omega), \omega \in \mathbb{P}^{p+1}\right\}, j=0, \ldots, p$, is a sequence of separable Gaussian processes distributed as $W_{j}^{\dagger}(\omega), j=0, \ldots, p$. Therefore, from (A.3),

$$
\begin{aligned}
\sup _{\omega \in I^{p+1}} \mid T^{-1 / 2} \sum_{t=1}^{T} R_{t}(\omega)-(p+1)^{-1 / 2} \sum_{j=0}^{p} & W_{j N}^{\dagger}(\omega) \mid \\
& =\sup _{\omega \in I^{p+1}}\left|(p+1)^{-1 / 2} \sum_{j=0}^{p}\left\{N^{1 / 2} S_{j N}(\omega)-W_{j N}^{\dagger}(\omega)\right\}\right|
\end{aligned}
$$




$$
\begin{aligned}
& \leqslant(p+1)^{-1 / 2} \sum_{j=0}^{p} \sup _{\omega \in I^{p+1}}\left|N^{1 / 2} S_{j N}(\omega)-W_{j N}^{\dagger}(\omega)\right| \\
& =\mathrm{O}\left\{N^{-1 / 2(p+2)}(\log N)^{3 / 2}\right\} \text { a.s. }
\end{aligned}
$$

Note that $W_{T}^{*}(\omega)=(p+1)^{-1 / 2} \sum_{j=0}^{p} W_{j N}^{\dagger}(\omega)$ is a separable Gaussian process with mean and covariances as $T^{-1 / 2} \sum_{t=1}^{T} \sum_{t}(\omega)$. That is, $E\left\{W_{T}^{*}(\omega)\right\}=0$, and for $\omega^{\prime} \in I^{p+1}$, $E\left\{W_{T}^{*}(\omega) W_{T}^{*}\left(\omega^{\prime}\right)\right\}=\Xi\left(\omega, \omega^{\prime}\right)$. From (A.4),

$$
\sup _{\omega \in r^{++1}}\left|\sum_{t=1}^{T} R_{t}(\omega)\right|=\mathrm{O}\left\{(T \log \log T)^{1 / 2}\right\} \text { a.s. }
$$

Then, Lemma 1, (A.5) and (A.6) show that

$$
\begin{gathered}
\sup _{\omega \in I^{p+1}}\left|T^{1 / 2} S_{T}(\omega)-W_{T}^{*}(\omega)\right|=\mathrm{O}\left\{T^{-1 / 2(p+2)}(\log T)^{3 / 2}\right\} \text { a.s. } \\
\sup _{\omega \in I^{p+1}}\left|T S_{T}(\omega)\right|=\mathrm{O}\left\{(T \log \log T)^{1 / 2}\right\} \text { a.s. }
\end{gathered}
$$

From (A.7) and (A.8), and using the same arguments as (4.57) in Csörgö (1979),

$$
\int_{\mathbb{R}^{p+1}} S_{T}^{2}(x) d\left\{\prod_{j=1}^{p+1} F_{1}\left(x_{j}\right)\right\} \stackrel{d}{\rightarrow} B \equiv \int_{p^{p+1}} W^{*}(\omega)^{2} d \omega .
$$

Applying the lemma in Kiefer (1959) as in Blum et al. (1961),

$$
B_{T}-\int_{\mathbb{R}^{p+1}} S_{T}^{2}(x) d\left\{\prod_{j=1}^{p+1} F_{1}\left(x_{j}\right)\right\}=\mathrm{o}_{\mathrm{p}}(1) .
$$

ProOF OF THEOREM 2. It suffices to prove that

$$
T^{-1} B_{T}-E\left\{S\left(Z_{1}\right)^{2}\right\} \rightarrow 0 \text { a.s. }
$$

which is proved from

$$
\begin{aligned}
T^{-1} \sum_{t=1}^{T}\left\{S_{T}\left(Z_{t}\right)^{2}-S\left(Z_{t}\right)^{2}\right\} & \rightarrow 0 \text { a.s. } \\
T^{-1} \sum_{t=1}^{T} S\left(Z_{t}\right)^{2}-E\left\{S\left(Z_{1}\right)^{2}\right\} & \rightarrow 0 \text { a.s. }
\end{aligned}
$$

Stute and Schumann's (1980) theorem proves (A.9) and (A.10) follows from $\left\{S(Z)_{t}^{2}\right.$, $t \geqslant 1\}$ ergodic.

ACKNOWLEDGEMENTS

This paper is based on research supported by the Spanish Dirección General de Investigación Científica y Técnica (DGICYT), reference number PB92-0247. I am grateful to Wenceslao González-Manteiga, Peter M. Robinson, Dag Tjøstheim, the associate editor and a referee for helpful comments. 


\section{REFERENCES}

BARTELS, R. (1982) The rank version of von Neumann's ratio test for randomness. J. Am. Statist. Assoc. 77, 40-46.

Blum, J. R., Kiefer, J. and Rosenblatt, M. (1961) Distribution free tests of independence based on the sample distribution function. Ann. Math. Stat. 32, 485-98.

Brillinger, D. R. (1969) An asymptotic representation of the sample df. Bull. Am. Math. Soc. 75 , 545- 47.

Brock, W., Dechert, D. and Scheinkman, J. (1987) A test for independence based on the correlation dimension. Preprint, University of Wisconsin.

Chan, N. H. and Tran, L. T. (1992) Nonparametric tests for serial dependence. J. Time Ser. Anal. $13,19-28$.

CotTerill, D. S. and Csörgö, M. (1985) On the limiting distribution of and critical values for the Hoeffding, Blum, Kiefer, Rosenblatt independence criterion. Stat. Decis. 3, 1-48.

Csörgö, M. (1979) Strong approximations of the Hoeffding, Blum, Kiefer, Rosenblatt multivariate empirical process. J. Multi. Anal. 9, 84-100.

Dufour, J.-M. (1981) Rank tests for serial dependence. J. Time Ser. Anal. 2, 118-28.

Edgington, E. S. (1987) Randomization Tests, 2nd Edn., New York: Dekker.

EFron, B. and Tibshirani R. J. (1993) An Introduction to the Bootstrap. London: Chapman and Hall.

FISHER, R. A. (1935) The Design of Experiments. Edinburgh: Oliver and Boyd.

Hallin, M., Ingenbleek, J.-F. and PuRI, M. L. (1985) Linear serial rank test for randomness against ARMA alternatives. Ann. Stat. 13, 1156-81.

Hallin, M., and PuRI, M. L. (1988) Optimal rank-based procedures for time series analysis: testing an ARMA model against other ARMA models. Ann. Stat. 15, 402-32.

HALlin, M. and MĖLARD, G. (1988) Rank based tests for randomness against first-order serial dependence. J. Am. Statist. Assoc. 83, 1117-22.

HoEFfDing, W. (1948) A nonparametric test of independence. Ann. Math. Stat. 19, 546-57.

KJEFER, J. (1959) $K$-samples analogues of the Kolmogorov-Smirnov and Cramér-v. Mises test. Ann. Math. Stat. 30, 4420-47.

KNOKE, J. D. (1977) Testing for randomness against autocorrelation: alternative tests. Biometrika $64,523-29$.

LJung, G. M. and Box, G. E. (1978) On a measure of lack of fit in time series models. Biometrika $65,297-303$.

Nadaraya, E. A. (1989) Nonparametric Estimation of Probability Densities and Regression Curves. Dordrech: Kluwer Academic Publishing.

Noreen, E. W. (1989) Computer Intensive Methods for Testing Hypotheses: an Introduction. New York: Wiley.

PINksE, C. A. P. (1993) A general characteristic function based measure applied to serial independence testing. Unpublished, London School of Economics.

RoBinson, P. M. (1991) Consistent nonparametric entropy-based testing. Rev. Econ. Stud. 58, 43753.

ROMANO, J. P. (1989) Bootstrap and randomization tests of some nonparametric hypotheses. Ann. Stat. 17, 141-59.

RosenblatT, M. (1975) A quadratic measure of deviation of two-dimensional density estimates and a test of independence. Ann. Stat. 3, 1-14.

Skaug, H. J. and TJøstheim, D. (1993) Nonparametric test for serial independence based on the empirical distribution function. Biometrika 80, 591-602.

Stute, W. and Schumann, G. (1980) A general Glivenko-Cantelli theorem for stationary sequences of random observations. Scand. J. Stat. 2, 102-4.

Whistler, D. E. N. (1990) Semi-parametric Models of Daily and Intra-Daily Exchange Rate Volatility. Unpublished Ph.D. Thesis, University of London. 